\title{
Hydrodynamics and Heat Transfer Characteristics of Free Surface Liquid Jet Impingement on a Convex Cylindrical Surface
}

\author{
Kuldeep Baghel $^{1 *}$, Arunkumar Sridharan', Janani Srree Muralidharan ${ }^{2}$ \\ ${ }^{1}$ Indian Institute of Technology Bombay \\ Powai, Mumbai, India \\ *Kuldeepb@iitb.ac.in; arunsri@iitb.ac.in; js.murallidharan@iitb.ac.in
}

\section{Extended Abstract}

A numerical study is presented to investigate free-surface liquid jet impingement on a uniformly heated semi-circular convex cylinder. In literature, air-jet impingement studies are available for both flat and curved surface configurations; however, only a few studies are available for free surface liquid jet impingement cases, and they are limited to the flat surface configurations. Present work numerically investigates heat transfer and hydrodynamic characteristics of free surface liquid jet impingement on a semi-cylindrical convex surface. The convex side of the semi-cylindrical surface is exposed to liquid jet. The interface is captured using the VOF method, and Scale Adaptive Simulation (SAS) is used to capture turbulence characteristics of the jet impingement phenomena. Jet emerges from a long circular pipe and strikes on a curved surface. Separate simulations are performed to get fully developed velocity and turbulence profiles at the nozzle exit, and these profiles are superimposed at the inlet of the existing domain for numerical simulation to serve as the initial condition for the simulations. Circular nozzle with $6 \mathrm{~mm}$ diameter is used in the simulations, and Reynolds number, which is defined based on the properties at the jet exit and characteristics length as nozzle exit diameter, is varied from 10000 to 27000 . The separation distance from the impingement surface to jet exit is fixed to nondimensional distance, $\mathrm{H} / \mathrm{d}=4$, where $\mathrm{H}$ is separation distance. Numerical results are compared with the experimental results of jet impingement on a curved surface obtained by the same author and have shown a good qualitative trend. The main objective of this work is to understand the effect of the curvature of the impingement surface on heat transfer. Five different curvature ratios (D/d) are used in the simulation. Curvature ratio is defined based on the diameter of the cylindrical surface (D) and the diameter of the circular nozzle (d). Heat transfer is characterized based on Nusselt number at the impingement surface. Nusselt number profiles at different locations on the impingement surface are compared for different curvature cases. Pressure, wall shear, velocity, and turbulence characteristics are presented for a few cases, and their relation to heat transfer is discussed.

It is found out that the effect of curvature on Nusselt number is more pronounced near the impact, and Nusselt number profiles are weakly dependent on curvature. With increasing curvature (D/d), Nusselt number near the impact is found to decreases. It is shown that the velocity gradient, which is affected by changing the curvature ratio near the impact are the primary reason for the difference in Nusselt number near the impact at the impingement surface. Negative pressure is also observed at the impingement surface at few locations. The significant outcome of the presented study is the weak dependency of Nusselt on curvature for D/d more than four. It is to investigate if (i) reduction in curvature ratio would facilitate better control of the effect of curvature on Nusselt number (ii) and if it would be significant. 